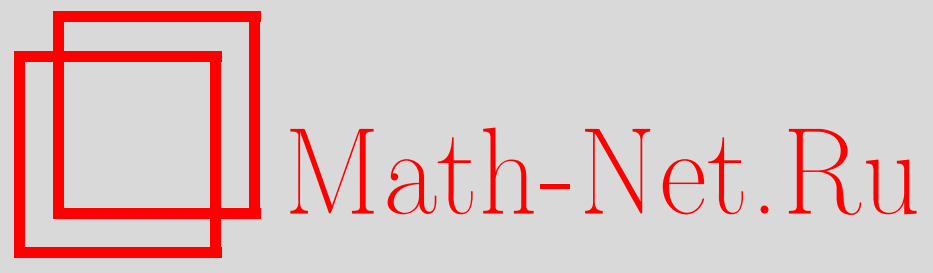

Н. А. Вирченко, М. А. Четвертак, Об одном обобщении функции Бесселя, Вестн. Сам. гос. техн. ун-та. Сер. Физ.-мат. науки, 2014, выпуск 4(), 1621

DOI: https://doi.org/10.14498/vsgtu1361

Использование Общероссийского математического портала MathNet.Ru подразумевает, что вы прочитали и согласны с пользовательским соглашением

http://www.mathnet.ru/rus/agreement

Параметры загрузки:

IP: 35.173 .219 .12

26 апреля 2023 г., 16:43:50

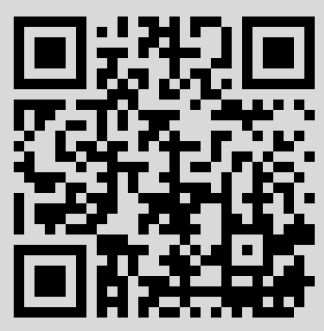


Вестн. Сам. гос. техн. ун-та. Сер. Физ.-мат. науки. 2014. № 4 (37). С. 16-21

ISSN: 2310-7081 (online), 1991-8615 (print) doi: http://dx.doi.org/10.14498/vsgtu1361

УДК 517.584, 517.923

\section{ОБ ОДНОМ ОБОБЩЕНИИ ФУНКЦИИ БЕССЕЛЯ}

\section{Н. А. Вирченко, М. А. Четвертак}

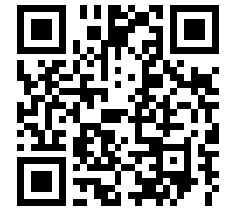

Национальный технический университет Украины

«Киевский политехнический институт»,

Украина, 03056, Киев, просп. Победы, 37.

\section{Аннотация}

Вводится обобщенная функция Бесселя $J_{\mu, \omega}(x)$ как одно из решений дифференциального уравнения

$$
x^{2} y^{\prime \prime}+x y^{\prime}+\left(x-\mu^{2}\right)\left(x+\omega^{2}\right) y=0, \quad \mu, \omega \notin \mathbb{Z} .
$$

Получено представление функции $J_{\mu, \omega}(x)$ в виде степенного ряда; получена и доказана теорема об интегральных представлениях этой функции. Изучены основные свойства этой функции; построено интегральное преобразование и доказана формула его обращения.

Ключевые слова: функция Бесселя, гипергеометрическая функция, интегральное преобразование.

doi: http://dx.doi.org/10.14498/vsgtu1361

Введение. Среди многообразия специальных функций особо выделяются функции Бесселя в силу своих многочисленных замечательных приложений в теории дифференциальных и интегральных уравнений, в физике, механике и др. (см., например библиографию в [1-9]). Функции Бесселя впервые возникли при рассмотрении задач о распостранении тепла в твердом круглом цилиндре, при исследовании колебаний растянутой круговой мембраны (работы Л. Эйлера), встречаются в работе Ж. Лагранжа по эллиптическим движениям. Систематическое изучение функций Бесселя начато Ф. Бесселем (1824 г.) в его научных работах.

Теперь функции Бесселя используются при решении широкого класса краевых задач математической физики, механики сплошных сред, в теории интегральных преобразований, так как они часто возникают при решении задач как прикладной, так и теоретической математики, в теории специальных функций и других отраслях прикладного естествознания [9-14].

1. Обобщенная функция Бесселя. Введем обобщенную функцию Бесселя $J_{\mu, \omega}(x)$ как одно из решений следующего дифференциального уравнения:

$$
x^{2} y^{\prime \prime}+x y^{\prime}+\left(x-\mu^{2}\right)\left(x+\omega^{2}\right) y=0,
$$

(C) 2014 Самарский государственный технический университет.

\section{Образец для цитирования}

Вирченко Н. А., Четвертак М. А. Об одном обобщении функции Бесселя // Вестн. Сам. гос. техн. ун-та. Сер. Физ.мат. науки, 2014. № 4(37). С. 16-21. doi: $10.14498 /$ vsgtu1361.

\section{Сведения об авторах}

Нина Афанасъевна Вирченко (д.ф.-м.н., проф.; nvirchenko@hotmail.com), профессор, каф. математического анализа и теории вероятностей.

Мария Александровна Четвертак (chetvertakmaria.math@gmail.com; автор, ведущий переписку), аспирант, каф. математического анализа и теории вероятностей. 
где $\mu, \omega \notin \mathbb{Z}$.

Заметим, что при $\mu^{2}=\omega^{2}=v$ уравнение (1) является известным уравнением Бесселя [1]:

$$
x^{2} y^{\prime \prime}+x y^{\prime}+\left(x^{2}-v^{2}\right) y=0
$$

Решение дифференциального уравнения (1) будем искать в виде

$$
y=\sum_{k=0}^{\infty} C_{k} z^{k+\rho}, \quad C_{0} \neq 0
$$

где $\rho$ - корень характеристического уравнения, $C_{k}$ - некоторые аналитические функции при всех $x$.

Подставив (2) в (1) и приравняв коэффициенты при одинаковых степенях $x$, получим следующую рекуррентную систему для определения $C_{k}$ :

$$
\begin{gathered}
(\rho+k)^{2} C_{k}+C_{k-2}+\left(\mu^{2}-\omega^{2}\right) C_{k-1}=0, \quad k=2,3, \ldots, \\
C_{2 m}=-\frac{C_{2 m-2}+\left(\omega^{2}-\mu^{2}\right) C_{2 m-1}}{(\rho+2 m)^{2}-\mu^{2} \omega^{2}}, \quad m=1,2, \ldots
\end{gathered}
$$

После преобразования (2) с учетом $C_{k}$, найденных из $(3),(4)$, получим

$$
y=J_{\mu, \omega}(x)=\left(\frac{x}{2}\right)^{\mu^{2}} \frac{1}{\Gamma\left(\frac{\mu^{2}+\mu \omega+2}{2}\right)} \cdot{ }_{1} F_{2}\left(1 ; \frac{\mu^{2}-\mu \omega+2}{2}, \frac{\mu^{2}+\mu \omega+2}{2} ;-\frac{x^{2}}{4}\right),
$$

где $\Gamma(x)$ - гамма-функция [2], ${ }_{1} F_{2}(a ; c, d ; x)$ - гипергеометрическая функция [15], определяемая следующим образом:

$$
{ }_{1} F_{2}(a ; c, d ; x)=\sum_{n=0}^{\infty} \frac{(a)_{n}}{(c)_{n}(d)_{n}} \frac{x^{n}}{n !},
$$

где $(a)_{n}$ - символ Похгаммера, определенный для $x \in \mathbb{C}$ и $n \in \mathbb{N}_{0}=\mathbb{N} \cup\{0\}$ :

$$
(a)_{0}=1, \quad(a)_{n}=a(a+1) \ldots(a+n-1), \quad n \in \mathbb{N} .
$$

Легко убедиться, что при $\mu^{2}=\omega^{2}=v$ функция, определяемая формулой $(5)$, совпадает с функцией Бесселя $J_{v}(x)[1]:$

$$
J_{v}(x)=\left(\frac{x}{2}\right)^{v} \frac{1}{\Gamma(v+1)} \cdot{ }_{0} F_{1}\left(v+1 ;-\frac{x^{2}}{4}\right) .
$$

\section{2. Интегральные представления функции $J_{\mu, \omega}(x)$.}

ТЕОРЕМА (ОБ ИНТЕГРАЛЬНЫХ ПРЕДСТАВЛЕНИЯХ ФУНКцИИ $J_{\mu, \omega}(x)$ ). При условиях существования функиии $J_{\mu, \omega}(x)$ справедливы следуюшие интегральнье представления:

$$
J_{\mu, \omega}(x)=\frac{\mu^{2}+\mu \omega}{2} \int_{0}^{1} \int_{0}^{1} \exp \left(-t \tau \frac{x^{2}}{4}\right)(1-t)^{\frac{\mu^{2}+\mu \omega}{2}-1}(1-\tau)^{\frac{\mu^{2}-\mu \omega}{2}} \tau^{-1} d t d \tau
$$




$$
\begin{aligned}
& J_{\mu, \omega}(x)=2\left(\mu^{2}+\mu \omega\right) \int_{0}^{\pi} \int_{0}^{\pi} \exp (-\left.\sin ^{2} \phi \sin \theta \frac{x^{2}}{4}\right)(\cos \phi)^{\mu^{2}+\mu \omega-1} \times \\
& \times(\cos \theta)^{\mu^{2}+\mu \omega+1} \sin \phi \sin ^{-1} \theta d \phi d \theta, \\
& J_{\mu, \omega}(x)=4 \int_{0}^{1} \int_{0}^{1} \exp \left(-\left(1-k^{2}\right)\left(1-l^{2}\right) \frac{x^{2}}{4}\right) \times \\
& \times k^{\mu^{2}+\mu \omega} l^{\mu^{2}-\mu \omega+1}\left(1-l^{2}\right)^{-1} d k d l .
\end{aligned}
$$

Доказ ательство формул (6)-(8) осуществляется при помощи соответствующих подстановок.

Учитывая для гипергеометрической функции ${ }_{1} F_{2}$ формулы

$$
\begin{aligned}
& { }_{1} F_{2}(1 ; k+m+1, r ; x)=(k+m) \Gamma(r) x^{\frac{1-r}{2}} \int_{0}^{1} I_{r-1}(2 \sqrt{x t}) t^{\frac{1-r}{2}}(1-t)^{k+m-1} d t, \quad(9) \\
& { }_{1} F_{2}(1 ; k+m+1, r ; x)=\frac{\Gamma(k+m+1)}{k ! \Gamma(m)} \int_{0}^{1}{ }_{1} F_{2}(1 ; m, r ; x t) t^{m-1}(1-t)^{k} d t,(10)
\end{aligned}
$$

где $I_{r-1}(x)$ - модифицированная функция Бесселя при $x \in \mathbb{C}$, можно получить еще ряд представлений для $J_{\mu, \omega}(x)$.

Заметим, что формулы (9), (10) доказываются непосредственной проверкой.

Используя представление функции $J_{\mu, \omega}(x)$ в виде ряда

$$
\begin{aligned}
& J_{\mu, \omega}(x)=\Gamma\left(\frac{\mu^{2}-\mu \omega}{2}+1\right) \times \\
& \quad \times \sum_{n=0}^{\infty} \frac{(-1)^{n}}{n !} \frac{1}{\Gamma\left(\frac{\mu^{2}-\mu \omega}{2}+n+1\right) \Gamma\left(\frac{\mu^{2}+\mu \omega}{2}+n+1\right)}\left(\frac{x}{2}\right)^{2 n+\mu^{2}},
\end{aligned}
$$

получаем формулу для дифференцирования:

$$
\frac{d}{d x} J_{\mu, \omega}(x)=A \sum_{n=0}^{\infty} \frac{(-1)^{n}}{n !} \frac{\left(2 n+\mu^{2}\right)}{2 \Gamma\left(v_{1}+n+1\right) \Gamma\left(v_{2}+n+1\right)}\left(\frac{x}{2}\right)^{2 n+\mu^{2}-1},
$$

где

$$
v_{1}=\frac{\mu^{2}-\mu \omega}{2}, \quad v_{2}=\frac{\mu^{2}+\mu \omega}{2}, \quad \mu^{2}=v_{1}+v_{2}, \quad A=\Gamma\left(\frac{\mu^{2}-\mu \omega}{2}+1\right) .
$$

3. Обобщенное интегральное преобразование. Введем обобщенное интегральное преобразование типа Бесселя:

$$
\left(I_{\mu, \omega} f\right)(x)=\int_{0}^{\infty} J_{\mu, \omega}\left(a ; c, d ;-\frac{x^{2}}{4} t\right) f(t) d t .
$$

Для простоты рассмотрим (11) в следующей форме:

$$
\left(I_{\mu, \omega} f\right)(x)=\int_{0}^{\infty} J_{\mu, \omega}(a ; c, d ;-x t) f(t) d t, \quad a, c, d \in \mathbb{C}, \quad \operatorname{Re} a>0 .
$$


Легко заметить, что (12) - интегральное $G$-преобразование [17]:

$$
(G f)(x)=\int_{0}^{\infty} G_{p, q}^{m, n}\left[\begin{array}{c|c}
\left(a_{i}\right)_{1, p} & x t \\
\left(\beta_{j}\right)_{1, q} & x
\end{array}\right] f(t) d t
$$

где $G_{p, q}^{m, n}-$ функция Мейера [16]:

$$
\begin{aligned}
& G_{p, q}^{m, n}\left[\begin{array}{l|l}
\left(a_{i}\right)_{1, p} & z \\
\left(\beta_{j}\right)_{1, q} &
\end{array}\right]= \\
& =\frac{1}{2 \pi i} \int_{L} \frac{\prod_{j=1}^{m} \Gamma\left(\beta_{j}+s\right) \prod_{i=1}^{n} \Gamma\left(1-a_{i}-s\right)}{\prod_{i=n+1}^{p} \Gamma\left(a_{i}+s\right) \prod_{j=m+1}^{q} \Gamma\left(1-\beta_{j}-s\right)} z^{-s} d s .
\end{aligned}
$$

С помощью интегрального преобразования Меллина получаем формулу обращения для (12). Справедлива следующая теорема.

Teоpema. Пусть $0<1-v<\operatorname{Re} a, \alpha_{0}=\max [1-\operatorname{Re} c, 1-\operatorname{Re} d]$. Ecлu $v>\alpha_{0}$, $\operatorname{Re} \lambda>v-1,(1-v)+\operatorname{Re}(a-c-d)=0, f \in L_{v, 2}$, mо имеет место следующая формула обрашения:

$$
f(x)=x^{-\lambda} \frac{d}{d x} x^{\lambda+1} \int_{0}^{\infty} G_{2,4}^{2,1}\left[\begin{array}{c|c}
-\lambda, 1-a \\
c-1, d-1,0,-\lambda-1
\end{array} \mid x t\right]\left({ }_{1} F_{2} f\right)(t) d t .
$$

\section{ORCID}

Нина Афанасьевна Вирченко: http://orcid.org/0000-0001-7229-0156

Мария Александровна Четвертак: http://orcid.org/0000-0003-4323-5101

\section{БИБЛИОГРАФИЧЕСКИЙ СПИСОК}

1. Watson G. N. A Treatise on the Theory of Bessel Functions. Cambridge: University Press, 1922. vi+804 pp.

2. Вирченко Н. А., Царенко В. Н. Дробные интегралъные преобразования гипергеометрического типа. Киев: Ин-т матем. НАН Украины, 1995. 216 с.

3. Волкодавов В. Ф., Канатников А. Н. Интегралъные преобразования и операционное исчисление. М.: МГТУ им. Баумана, 1968. 228 с.

4. Галицын А. С., Жуковский А. Н. Интегралъные преобразования и специалъные функции в задачах теплопроводности. Киев: Наукова думка, 1986. 284 с.

5. Диткин В. А., Прудников А. П. К теории операционного исчисления, порожденного уравнением Бесселя // Ж. вычисл. матем. и матем. физ., 1963. Т. 3, № 2. С. 223-238.

6. Коренев Б. Г. Введение в теорию бесселевых функиий. М.: Наука, 1971. 288 с.

7. Лебедев Н. Н. Специалъные функи,и и их приложения. М.: Физматгиз, 1963. 358 с.

8. Никифоров А. Ф., Уваров В. Б. Специалъные функции математической физики. М.: Наука, 1978. $320 \mathrm{c}$.

9. Самко С. Г., Килбас А. А., Маричев О. И. Интегралы и производные дробного порядка и некоторые их приложения. Минск: Наука и техника, 1987. 688 с.

10. Bonilla B., Kilbas A. A., Rivero M., Rodriguez L., Trujillo J. J. Modified Bessel-type function and solution of differential and integral equations // Indian J. Pure and Appl. Math., 2000. vol. 31, no. 1. pp. 93-109.

11. Kalla S. L., Virchenko N., Tsarenko V. On some fractional order integral transforms generated by orthogonal polynomials // Applied Mathematics and Computation, 1998. vol. 91, no. 2-3. pp. 209-219. doi : 10.1016/s0096-3003(97) 10019-4.

12. Khajan N. G. A modified finite Hankel transforms// Integral Transforms and Special Functions, 2003. vol. 14, no. 5. pp. 403-412. doi : 10.1080/10652460310001600654. 
13. Virchenko N., Kalla S. L., Zaikina S. On some generalized integral transforms // Hadronic J., 2009. vol. 32 , no. 5. pp. 539-548.

14. Virchenko N. On one effective method of solving of mixed boundary value problems / Abstracts of International Congress of Mathematicians. Zurich, 1994. pp. 224.

15. Erdélyi A., Magnus W., Oberhettinger F., Tricomi F. G. Higher transcendental functions. vol. 1 / Bateman Manuscript Project. New York: McGraw-Hill Book Co., 1953. xxvi+302 pp.

16. Kilbas A. A., Saigo M. H-Transforms. Theory and Applications / Analytical Methods and Special Functions. Boca, Raton, etc.: CRC Press, 2004. doi : 10.1201/9780203487372.

17. Вирченко Н. А. Об интегральном преобразовании с обобщенной функцией гипергеометрического типа / Труды Двенади,атой межвузовской конферениии (29-31 мая 2002 г.). Часть 3: Дифференциальные уравнения и краевые задачи / Матем. моделирование и краев. задачи. Самара: СамГТУ, 2002. С. 125.

Поступила в редакцию 03/XI/2014;

в окончательном варианте - 26/XI/2014;

принята в печать $-27 / \mathrm{XI} / 2014$.

Vestn. Samar. Gos. Techn. Un-ta. Ser. Fiz.-mat. nauki

[J. Samara State Tech. Univ., Ser. Phys. \& Math. Sci.] 2014. Issue 4 (37). Pp. 16-21

ISSN: 2310-7081 (online), 1991-8615 (print)

doi: http://dx.doi.org/10.14498/vsgtu1361

MSC: 33C10, 34B30

\section{ON ONE GENERALIZATION OF BESSEL FUNCTION}

\section{N. A. Virchenko, M. O. Chetvertak}

National Technical University of Ukraine "Kiev Polytechnic Institute",

37, Peremogi st., Kiev, 03056, Ukraine.

\section{Abstract}

In this paper the generalized Bessel function $J_{\mu, \omega}(x)$ is introduced. The function $J_{\mu, \omega}(x)$ is given as one solution of the following differential equation:

$$
x^{2} y^{\prime \prime}+x y^{\prime}+\left(x-\mu^{2}\right)\left(x+\omega^{2}\right) y=0, \quad \mu, \omega \notin \mathbb{Z} .
$$

The representation of the $J_{\mu, \omega}(x)$ by the power series is given. The theorem on integral representations of the function $J_{\mu, \omega}(x)$ is established. The main properties of the function $J_{\mu, \omega}(x)$ are studied. The integral transforms of Bessel type with the function $J_{\mu, \omega}(x)$ is constructed. Formula of inversion of this transform is received.

Keywords: Bessel function, hypergeometric function, integral transform.

doi: http://dx.doi.org/10.14498/vsgtu1361

(C) 2014 Samara State Technical University.

\section{How to cite Reference}

Virchenko N. A., Chetvertak M. O. On one generalization of Bessel function, Vestn. Samar. Gos. Tekhn. Univ., Ser. Fiz.-Mat. Nauki [J. Samara State Tech. Univ., Ser. Phys. \& Math. Sci.], 2014, no. 4(37), pp. 16-21. doi: 10.14498/vsgtu1361. (In Russian)

\section{Authors Details}

Nina A. Virchenko (Dr. Phys. \& Math. Sci.; nvirchenko@hotmail.com), Professor, Dept. of Mathematical Analysis and Probability Theory.

Maria O. Chetvertak (chetvertakmaria.math@gmail.com; Corresponding Author), Postgraduate Student, Dept. of Mathematical Analysis and Probability Theory. 


\section{ORCID}

Nina A. Virchenko: http://orcid.org/0000-0001-7229-0156

Maria O. Chetvertak: http://orcid.org/0000-0003-4323-5101

\section{REFERENCES}

1. Watson G. N. A Treatise on the Theory of Bessel Functions. Cambridge, University Press, 1922, vi+804 pp.

2. Virchenko N. A., Tsarenko V. N. Drobnye integral'nye preobrazovaniia gipergeometricheskogo tipa [Fractional integral transformations of hypergeometric type]. Kiev, In-t matem. NAN Ukrainy, 1995, 216 pp. (In Russian)

3. Volkodavov V. F., Kanatnikov A. N. Integral'nye preobrazovaniia i operatsionnoe ischislenie [Integral Transforms and Operational Calculus]. Moscow, Bauman MSTU, 1968, 228 pp. (In Russian)

4. Galitsyn A. S., Zhukovskii A. N. Integral'nye preobrazovaniia $i$ spetsial'nye funktsii $v$ zadachakh teploprovodnosti [Integral transforms and special functions in heat conduction problems]. Kiev, Naukova Dumka, 1986, 284 pp. (In Russian)

5. Ditkin V.A., Prudnikov A. P. On the theory of the operational calculus for the Bessel equation, U.S.S.R. Comput. Math. Math. Phys., 1963, vol.3, no.2, pp. 296-315. doi: 10. 1016/0041-5553(63) 90022-3.

6. Korenev B. G. Vvedenie v teoriiu besselevykh funktsii [Introduction to the theory of Bessel functions]. Moscow, Nauka, 1971, 287 pp. (In Russian)

7. Lebedev N. N. Spetsial'nye funktsii $i$ ikh prilozheniia [Special functions and their applications]. Moscow, Fizmatgiz, 1963, 358 pp. (In Russian)

8. Nikiforov A. F., Uvarov V. B. Spetsial'nye funktsii matematicheskoi fiziki [Special functions of mathematical physics]. Moscow, Nauka, 1978, 320 pp. (In Russian)

9. Samko St. G., Kilbas A. A., Marichev O. I. Fractional integrals and derivatives: theory and applications. New York, NY, Gordon and Breach, 1993, xxxvi+976 pp.

10. Bonilla B., Kilbas A. A., Rivero M., Rodriguez L., Trujillo J. J. Modified Bessel-type function and solution of differential and integral equations, Indian J. Pure and Appl. Math., 2000, vol. 31, no. 1, pp. 93-109.

11. Kalla S. L., Virchenko N., Tsarenko V. On some fractional order integral transforms generated by orthogonal polynomials, Applied Mathematics and Computation, 1998, vol.91, no. 2-3, pp. 209-219. doi: 10.1016/s0096-3003(97)10019-4.

12. Khajan N. G. A modified finite Hankel transforms, Integral Transforms and Special Functions, 2003, vol.14, no. 5, pp. 403-412. doi: 10.1080/10652460310001600654.

13. Virchenko N., Kalla S. L., Zaikina S. On some generalized integral transforms, Hadronic J., 2009, vol. 32, no. 5, pp. 539-548.

14. Virchenko N. On one effective method of solving of mixed boundary value problems, Abstracts of International Congress of Mathematicians. Zurich, 1994, pp. 224.

15. Erdélyi A., Magnus W., Oberhettinger F., Tricomi F. G. Higher transcendental functions, vol. 1, Bateman Manuscript Project. New York, McGraw-Hill Book Co., 1953, xxvi+302 pp.

16. Kilbas A. A., Saigo M. H-Transforms. Theory and Applications, Analytical Methods and Special Functions. Boca, Raton, etc., CRC Press, 2004. doi : 10.1201/9780203487372.

17. Virchenko N. A. On an integral transform with generalized hypergeometric function, Proceedings of the Twelfth Inter-University Conference. Part 3, Matem. modelirovanie i kraev. zadachi. Samara, Samara State Technical Univ., 2002, pp. 125 (In Russian).

Received 03/XI/2014;

received in revised form $26 / \mathrm{XI} / 2014$;

accepted $27 / \mathrm{XI} / 2014$. 